\title{
Integrated Data Analysis for Fusion: A Bayesian Tutorial for Fusion Diagnosticians
}

\author{
Andreas Dinklage ${ }^{1}$, Heiko Dreier ${ }^{1}$, Rainer Fischer ${ }^{2}$, Silvio Gori ${ }^{2}$, \\ Roland Preuss ${ }^{1}$ and Udo von Toussaint ${ }^{2}$
}

\author{
Max-Planck-Institut für Plasmaphysik \\ EURATOM Association \\ ${ }^{1}$ Wendelsteinstr. 1, 17491 Greifswald, Germany \\ ${ }^{2}$ Boltzmannstr. 2, 85748 Garching, Germany
}

\begin{abstract}
Integrated Data Analysis (IDA) offers a unified way of combining information relevant to fusion experiments. Thereby, IDA meets with typical issues arising in fusion data analysis. In IDA, all information is consistently formulated as probability density functions quantifying uncertainties in the analysis within the Bayesian probability theory. For a single diagnostic, IDA allows the identification of faulty measurements and improvements in the setup. For a set of diagnostics, IDA gives joint error distributions allowing the comparison and integration of different diagnostics results. Validation of physics models can be performed by model comparison techniques. Typical data analysis applications benefit from IDA capabilities of nonlinear error propagation, the inclusion of systematic effects and the comparison of different physics models. Applications range from outlier detection, background discrimination, model assessment and design of diagnostics. In order to cope with next step fusion device requirements, appropriate techniques are explored for fast analysis applications.
\end{abstract}

Keywords: magnetic confinement fusion, data analysis, data fusion, integrated data analysis, Bayesian probability theory

PACS: 02.50.Cw, 07.05.Fb, 07.05.Mh, 52.70.-me

\section{DATA ANALYSIS FOR MAGNETIC CONFINEMENT FUSION DEVICES}

Next step fusion devices define several new requirements for data analysis. Since the demonstration of fusion reactor capabilities aims at steady-state operation, the data processing paradigm needs to be shifted from a shoot-and-collect to a view-and-react philosophy. Therefore, fast and reliable physics information is mandatory to use the experimental devices most efficiently and off-line analysis schemes as in pulsed devices will restrict operational capabilities. Long-time scales due to plasma-wall effects and magnetic field relaxation will require intelligent control schemes which employ physical models in multi-variate adaptive controllers.

The major challenge for data analysis in magnetic fusion experiments is to link many different heterogeneous information sources [1]. The reason for the heterogeneity lies in the variety of methods in plasma diagnostics which is required to 
determine the parameters for the assessment of device performance. In engineering, e.g. robotics, this link of different signals is called data fusion [2] which aims at reduction of uncertainties by a synergistic combination of multiple measurements. The task for fusion data analysis goes even one step ahead since the link of data requires extensively the use of physical models, e.g. equilibrium calculations or transport considerations.

Data analysis in fusion poses several system specific issues. The large number of intricate dependencies makes magnetic confinement fusion devices (MCF) a complex, large system. The complexity is even enhanced by the fact that the information suffers from heterogeneity not only in space and time but particularly in quality. In consequence, the validation of data is a demanding and time consuming task. Any progress towards an accelerated data analysis process allows one to increase both the number of analyzed fusion experiments and the quality of analyses. A vision is a framework aiming at automated procedures for analyses, validation and the detection of new features (e.g. pattern recognition techniques or data mining). In addition to an increased efficiency for an off-line analysis, the potential benefit of advanced data analysis methods is to allow a direct feed-back both to device operation (see e.g. [3]) and the planning of experiments.

Present data analysis methods fail to meet with these requirements for large fusion devices in general. For burning plasma experiments, new data analysis issues resulting from deterioration of signals in harsh environments may even complicate the analysis task. The key issue why data fusion in MCF devices is troublesome is the lack of standardization of uncertainty measures. Uncertainties are the key for a quantitative assessment of different data - the error is the information which allows to put decisions on agreement of data or to indicate disagreements and failures.

A concept to meet with typical fusion data analysis issues and new data analysis requirements is the Integrated Data Analysis (IDA). From an information science point of view, data fusion in IDA employs Bayesian Probability Theory (BPT). The strict formulation of the data analysis problem within BPT is the required data analysis standard. Moreover, the first-principle based calculus of BPT allows a couple of further applications typically occurring in fusion science ranging from outlier detection, background estimation to model selection for confinement studies. Although the thorough assessment of uncertainties and the technical treatment of high dimensional integrals lead to substantial effort in the data analysis process, techniques for fast analysis are available as well: Bayesian Neural Networks can be used for fast implementations of complex analysis tasks since the Bayesian pruning algorithm leads to efficient networks. Moreover, the proposed data analysis recipe allows for tools for the design of diagnostics and the planning of experiments.

This paper introduces the IDA concept after a brief revision of some data analysis requirements. Examples for applications are introduced. 


\section{Survey of Data Analysis Requirements}

Issues, resulting requirements and possible IDA techniques to cope with the requirement are summarized in Table 1, particularly including issues from steady-state devices and harsh environments. The purpose of this list is to give a survey on the present requirement discussion and to arrive at a prioritization of tasks.

Table 1: Data Analysis Requirements for Fusion Devices.

\begin{tabular}{|c|c|c|}
\hline Issue & Requirement & $\begin{array}{c}\text { Integrated Data Analysis } \\
\text { Technique }\end{array}$ \\
\hline Data Analysis Standard & $\begin{array}{c}\text { Framework for standardized } \\
\text { data analysis }\end{array}$ & $\begin{array}{c}\text { Standardized Bayesian error } \\
\text { treatment }\end{array}$ \\
\hline Global data consistency & $\begin{array}{c}\text { - standardized error analysis } \\
\text { - coherent linkage of different } \\
\text { data analysis procedures } \\
\text { - linkage to modeling }\end{array}$ & $\begin{array}{c}\text { Bayesian formulation } \\
\text { (sum and product rule for pdfs) }\end{array}$ \\
\hline Systematic errors & $\begin{array}{l}\text { - error propagation for model } \\
\text { parameters }\end{array}$ & hyper parameter, marginalization \\
\hline Amount of data & Automated analyses & $\begin{array}{c}\text { Bayesian formulation (standard) } \\
\text { Bayesian Neural Networks }\end{array}$ \\
\hline Reactor like operation & On-line physics analysis & $\begin{array}{c}\text { Laplace approximations, BNN, } \\
\text { IDA }\end{array}$ \\
\hline Data mining & $\begin{array}{l}\text { Novelty detections } \\
\text { Data warehousing }\end{array}$ & $\begin{array}{c}\text { BNN, probability density } \\
\text { functions, standard formulation }\end{array}$ \\
\hline $\begin{array}{l}\text { Error propagation with } \\
\text { interdependencies/parameter } \\
\text { correlations }\end{array}$ & $\begin{array}{c}\text { - generalized error propagation } \\
\text { - treatment of non-Gaussian } \\
\text { errors }\end{array}$ & $\begin{array}{c}\text { Bayesian formulation, } \\
\text { Integrated Data Analysis }\end{array}$ \\
\hline Sensitivity optimization & Super-Fit (joint analysis) & Integrated Data Analysis \\
\hline Model choice & Technique for model comparison & Bayesian model comparison \\
\hline $\begin{array}{l}\text { Direct model assessment (e.g. } \\
\text { data in transport models) }\end{array}$ & $\begin{array}{c}\text { - inference of derived quantities } \\
\text { (e.g. gradients) }\end{array}$ & $\begin{array}{c}\text { Bayesian formulation } \\
\text { (marginalization, forward } \\
\text { model) }\end{array}$ \\
\hline Signal identification & Signal - background separation & Mixture models \\
\hline Radiation induced effects & outlier identification & $\begin{array}{c}\text { Mixture models, } \\
\text { Robust fitting (non-Gaussian } \\
\text { likelihood) }\end{array}$ \\
\hline $\begin{array}{l}\text { Use of physics data for device } \\
\text { control }\end{array}$ & Fast analyses for real-time use & $\begin{array}{c}\begin{array}{c}\text { Simplified/adapted data analysis } \\
\text { models } \\
\text { Bayesian Neural Networks }\end{array} \\
\end{array}$ \\
\hline Identification of transient effects & $\begin{array}{l}\text { Combination of data for in-situ } \\
\text { calibration }\end{array}$ & Bayesian formulation \\
\hline Planning of experiments & $\begin{array}{c}\text { Optimized set of control } \\
\text { parameters for experimental } \\
\text { settings }\end{array}$ & Bayesian Experimental Design \\
\hline Diagnostic Design & Optimization of settings & Bayesian Experimental Design \\
\hline $\begin{array}{l}\text { Robust/redundant set of } \\
\text { diagnostics in harsh } \\
\text { environments }\end{array}$ & $\begin{array}{l}\text { Multi-tasking diagnostics, } \\
\text { Use of information in case of } \\
\text { failures }\end{array}$ & $\begin{array}{l}\text { Meta-Diagnostics } \\
\text { Integrated Analysis }\end{array}$ \\
\hline $\begin{array}{l}\text { Identification of failures \& } \\
\text { mismeasurements }\end{array}$ & validation tools & $\begin{array}{l}\text { Probability density function } \\
\text { Interdependencies (IDA) }\end{array}$ \\
\hline
\end{tabular}




\section{BASIC CONCEPTS}

Bayesian Probability Theory is introduced in several textbooks, see e.g. [4,5]. Here, we give a summary introducing the basic concepts and notions.

Data analysis is regarded to be a learning process: knowledge (or ignorance) on a parameter of interest is updated with knowledge from measurements. Mathematically, the updated knowledge on a parameter $q$ due to data $d$ can be phrased in a conditional probability

$$
p(q \mid d, I)
$$

which reads to be the conditional probability $p$ to find the parameter $q$ given the data $d$. By explicitly mentioning the background information $I$, it is acknowledged that information is always context sensitive. Both the quantity and the data can be vectors. In the Bayesian concept $p$ encodes the uncertainty on the quantity $q$.

The calculus of conditional probabilities is based on the sum and the product rule for probabilities. Bayes theorem results from product rule:

$$
p(q \mid d, I)=\frac{p(d \mid q, I) \times p(q \mid I)}{p(d \mid I)}
$$

where $I$ is the background of the prior information $p(q \mid I)$ on the quantity $q$, i.e., the knowledge on $q$ without data $d$. IDA uses the prior to encode physics or technical knowledge, e.g. positivity of temperatures or detection limits. In order to describe the measurement, the likelihood function $p(d \mid q, I)$ describes the probability to find data $d$ given a quantity $q$ under background information $I$. The likelihood function employs the forward calculation and the error statistics of the measurement. The evidence $p(d \mid I)$ ensures that the sum of all possible outcome of $q$ is normalized. The actual result $p(q \mid d, I)$ is called the posterior probability. It represents the update from $p(q \mid I)$ with data $d$. Hence, Bayes theorem (2) provides the prescription for learning from data, i.e. the transformation from a less informative state to a more informative one.

\section{THE BAYESIAN RECIPE}

In the Bayesian formulation the course of actions in data analysis (parameter estimation) is:

- a clear statement of the data analysis problem, i.e. the forward calculation from the quantity of interest to the data and the background (context) information. 
- formulation of a data model (likelihood) with a quantification of all uncertainties (provided by experimental assessments or by physically justified assumptions, e.g. densities have to be positive)

- quantification of the prior information

- the inference step: the application of Bayes theorem

- the focusing step: marginalization onto the quantity of interest.

The final steps are an application of the product and sum rules. The marginalization is a technique to derive estimates of quantities from the full posterior: the posterior contains all parameters which enter the forward model like, e.g., calibration factors $c_{i}$ as well as quantities to be inferred, e.g. densities $n\left(q=\left\{c_{i}, n\right\}\right)$. For the estimation of the specific quantity of interest, one to apply the marginalization rule to extract the desired information from the full posterior, in this example:

$$
p(n \mid d, I)=\int p\left(n, c_{i} \mid d, I\right) d c_{i}
$$

The result of Eq. (3) is the marginal posterior of quantity $n$. Since Eq. (3) incorporates the uncertainty of ${ }^{c_{i}}$ in the estimation of $n$, the marginalization is a generalization of Gaussian error propagation laws.

These two basic rules can be applied both for the analysis of a single diagnostics and for a set of diagnostics: independent probabilities need to be simply multiplied. Therefore, the rules (2) and (3) are the framework of an integrated data analysis.

Parameter estimation, as discussed, is one of the basic problems in data analysis. A second basic problem in data analysis is the question if a model or hypothesis should be preferred to a different one. This problem is called model comparison. In the Bayesian framework this can be treated by regarding the 'quantity of interest' to be a hypothesis or a model. A typical example is the question if a polynomial of nth degree fits data better than one of $(n+1)^{\text {th }}$ degree. In usual approaches, one has to put a decision on the trade-off between model complexity (which generally leads to lower discrepancies between data and fits) and model simplicity (which is the question if a lower complex model already explains the data within the error margins of the experiment). Bayesian model comparison allows one to compare the probability of different models ${ }^{M_{i}}$ which is quantified by the odds ratio $\mathrm{O}$ :

$$
O=\frac{p\left(M_{i} \mid d, I\right)}{p\left(M_{j} \mid d, I\right)} \text {. }
$$


If models are not to be preferred a priori, the odds ratio can be rephrased in the ratio of global likelihoods [4] which give the probability to find the data if a model is given. Since this quantity includes integration over the model parameter space, a higher complexity is punished by weighting the enlarged parameter space. This principle is called Occam's razor which states that a simpler model is to be preferred when it explains the data. It is clear, that a measure for model consistency must incorporate errors (as done in Eq. (4)), since those allow one to judge if deviances are significant.

A third basic problem is the design of measurements, i.e. which settings are to be chosen to optimize the outcome of an experiment. In the Bayesian experimental design [6] it is proposed to employ a utility function. A utility function based on information theory is the Kullback-Leibler distance [7] which measures the information gain of a measurement by comparing the posterior probability with the probability without a measurement:

$$
U_{K L}=\int p(\theta \mid d, I) l d(p(\theta \mid d, I) / p(\theta \mid I)) d \theta
$$

Marginalizing the utility function over an expected data space yields an expected utility $E U$ which depends on the settings $\eta$ only:

$$
E U(\eta)=\int p(D \mid \eta, I) U d D
$$

The expected utility can be maximized with respect to $\eta$ in order to arrive at the requested optimum setting.

\section{IDA OF SINGLE DIAGNOSTICS: FROM ONE ...}

An example for the application of IDA is the analysis of Thomson scattering data on Wendelstein 7-AS [8]. As usual for Thomson scattering, the purpose of the measurement is the determination of electron densities and temperatures. Background information is given by the details of the experimental set-up, calibration measurements and so on. The forward function is given by the data descriptive equation of the measurement:

$$
D_{T h}^{(i, j)}=c_{\text {geom }}^{(i)} P_{i} n_{e}^{(i)} \sigma_{T h} \int \tau^{(i, j)}(\lambda) S\left(\lambda, T_{e}^{(i)}, \Theta^{(i)}\right) d \lambda
$$

The data model considers background radiation, amplifications and all respective noise sources. For the specific formulation of the likelihood and prior probability density functions one needs to assess all parameters $\vec{\eta}$ entering the model, a good guidance is given by the full data model which transfers the physics origins of the measurement to the data as measured. The likelihood for data $d$ measured with an error $\sigma$ is given by the normal distribution according to the principle of maximum entropy, i.e., the most uninformative likelihood function for given data and errors:

$$
p\left(d \mid n_{e}, T_{e}, \vec{\eta}, \sigma, I\right)=\frac{1}{\Pi_{1}^{n} \sqrt{2 \pi} \sigma_{k}} \exp \left(-\frac{1}{2} \sum\left(\frac{d_{k}-D_{k}\left(n_{e}, T_{e}, \vec{\eta}\right)}{\sigma_{k}}\right)^{2}\right)
$$


Here, the likelihood function resembles the $\chi^{2}$ misfit statistics in accordance with the maximum entropy principle [5]. For the priors on density and temperature, flat functions cut at high values were chosen (e.g. $25 \mathrm{meV}<T_{e}<10 \mathrm{keV}$ ).

It should be noted that the largest effort in the application of the Bayesian recipe lies in the specification of all parameters and errors. The formalized treatment requires numerical techniques for high-dimensional integration.

The Bayesian analysis was found to have a couple of important benefits meeting with fusion specific requirements discussed in the introduction. Moreover, the Integrated Data Analysis formulation can be proposed to serve as a data analysis standard. Then, a reliable comparison of results can be performed. Second, the result contains all (non-linear) correlations introduced by the forward model. In the case of Thomson scattering, a correlation of electron temperature and density is introduced by Eq. (7). As will be discussed later, these correlations are informative and are barely used in traditional data analysis approaches. Third, the sensitivity of the Thomson scattering analysis in W7-AS was enhanced. This was of particular interest for formerly unexpected high density H-mode regimes with very high densities. Fourth, systematic effects can be included in the Bayesian approach by additional model parameters. These model parameters are assigned with an appropriate prior distribution $[4,5]$. In the case of the Thomson scattering analysis alignment factors were introduced describing differences in the optical path in laboratory calibrations and at the plasma vessel. The technique to treat these systematic effects is marginalization. Fifth, the data model can be used to assess the impact of different errors (e.g. systematic alignment errors or statistical noise due to electronics). This assessment is valuable for the design and maintenance of diagnostics.

\section{... TO MANY: INTEGRATED ANALYSIS OF DIAGNOSTICS SETS}

The step to an integrated data analysis is conceptually straight-forward once diagnostic models are available. Provided the data are taken independently - a trivial prerequisite for separate measurements - the linked result is proportional to the joint product of likelihoods and prior probabilities. IDA infers the quantities inductively (calculation of the probability to find a quantity, cf. Fig. 1(b)), differently to traditional approaches which perform a deductive analysis (Fig. 1(a). Fig. 1 shows the concept for a combination of Thomson scattering and ECE. The traditional approach requires iterative validation steps, each of which individually affected by additional information. This leads to complicated validation steps which require large effort for documentation. The IDA standardized formulation is compact and transparent. The joint posterior is the result of the integrated analysis. 


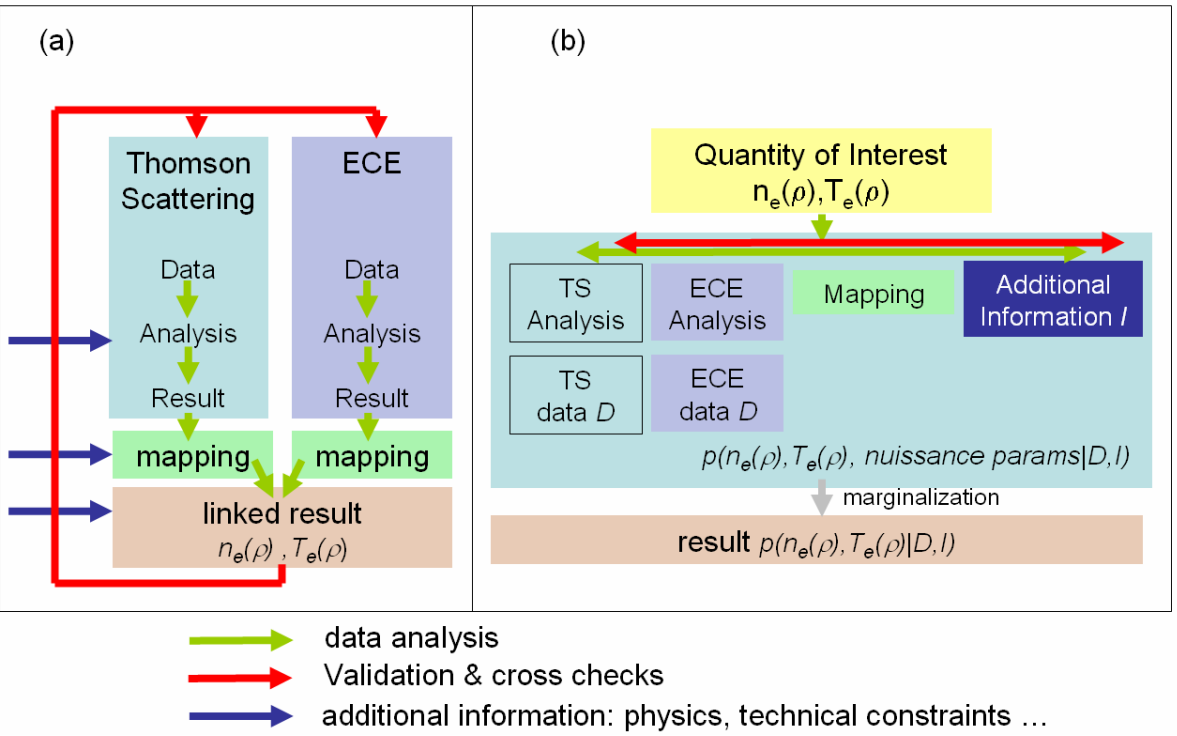

FIGURE 1. Traditional (a) vs. IDA (b) data fusion (combination of Thomson scattering and ECE).

The feasibility of this approach was demonstrated in Ref. [9]. The results show the power of the integrated approach with respect to the reduction of uncertainties of combined analyses: the joint (integrated) probability density function can be regarded as a meta-diagnostic which takes its (heterogeneous) data from different diagnostic units. This approach allows one to combine the measuring capabilities of different diagnostics to make use of their redundancies. An application of this idea is relevant to diagnostics in harsh environments where set-ups might suffer from radiation effects or from a lack of accessibility for maintenance.

A technical representation of IDA is given by probabilistic graphical models [10]. IDA can be used to evaluate uncertainties of formerly not accessible quantities, e.g. the error of the equilibrium (mapping). IDA also allows one to use physics constraints. Fig. 2(a) shows a flow diagram of already analyzed data, fits and model quantities; the link of which is given by the arrows representing probabilistic or deterministic relationships. In the analysis, the data are $n_{e}, T_{e}, T_{i}$ and $E_{r}$ measurements fitted to smooth model functions. Those are used as input for equilibrium calculations and for transport analyses. The latter provides a link of density and temperature profiles to the radial electric field. Moreover, the particle $\Gamma$ and heat fluxes $Q$ are outcome of this analysis and can be compared with results from separate modeling results (solid curves in Fig. 2 (b)). The joint posterior can be regarded as a super-fit to all data which is shown in Fig. 2(b) [11]. The uncertainty of the fit is indicated by the grey shaded histograms in Fig. 2(b). 

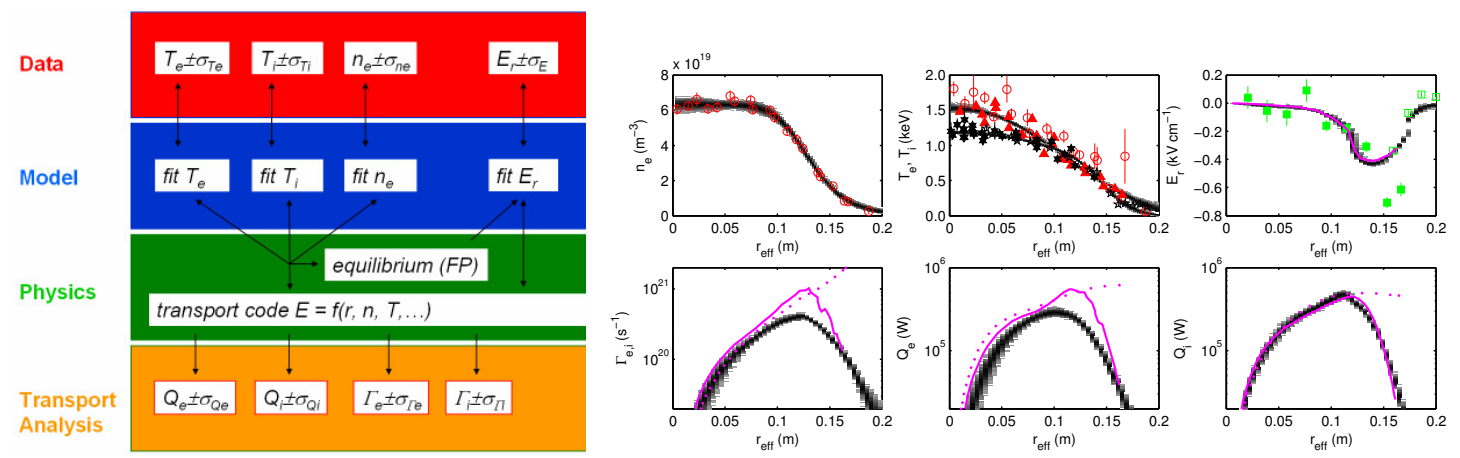

FIGURE 2. The left panel shows information sources and the data fusion scheme for a super-fit including equilibrium calculations and transport equations to link, e.g. data for the radial electric field. The right panel shows the result; note the errors in the quantities from the transport analysis.

\section{MORE APPLICATIONS OF BAYESIAN PROBABILITY THEORY IN FUSION}

In addition to conceptual improvements of the data fusion issue for fusion, Bayesian probability gives several solutions to fusion typical problems. The following selection gives some examples.

The idea to model systematic effects by additional model parameters can be extended to data descriptions consisting of two mutual exclusive hypotheses. An example is a typical spectroscopic issue: does a data point belong to a peak or to background? Mixture models consist of a sum representing the exclusive propositions and the occurrence of which are assigned to their probability: the probability to find a datum consist of the probability to find the datum to be a spectral line and the probability, that the datum is not a spectral line. In the introduced terminology, this probability is a hyper-parameter which can be marginalized. This technique was successfully applied to background estimation [12] and outlier estimation [13].

Rather than inferring parameters of the data model, the Bayesian framework may also be employed for the design of fusion measurements [14, 15]. The design is considered to provide experimental settings to measure given quantities of interest optimally as suggested in the previous chapter. So far, optimization studies of interferometer chords and Thomson scattering spectral filters were performed. Physically motivated optimization targets can be defined and cost-vs.-efficacy considerations can be performed on the basis of information gain measures. The outcome indicates the impact of errors on the optimum configuration. An application of the idea to planning of experiments is underway.

The model comparison technique can be used to test different basic plasma models against confinement data. The idea consists in the application of the Kadomtsev/Connor-Taylor invariance principle: If a basic equation is invariant against scaling transformation, then any derived relationship is invariant again. An example is basic plasma equations (e.g. Fokker-Planck kinetic equation) and energy confinement scaling laws. Model differences were found, e.g., in different $\beta$ regimes of stellarator confinement data [16]. 
Artificial neural networks have several applications. A key problem is the so-called pruning, i.e. to reduce the neural network to relevant parts. Here, Bayesian neural networks (BNN) employ model comparison techniques to identify the relevant parts of the network. Again, errors play a key role in this consideration. BNNs also allow the identification of novelties since confidence measures for the reconstructed quantities are part of the BNN result [17].

\section{SUMMARY AND OUTLOOK}

The concept of integrated data analysis is a framework meeting with many data analysis issues of contemporary fusion devices. IDA also faces new challenges for next step fusion machines. The applications of the IDA concept indicate feasibility and the data analysis techniques were implemented in routine operation and applied to online data analyses. IDA offers a frame-work for standardized data-analysis.

\section{ACKNOWLEDGMENTS}

This paper is dedicated to Prof. V. Dose who introduced the author to the Bayesian variant. This work was partially supported by Deutsche Forschungsgemeinschaft through Sfb TR24.

\section{REFERENCES}

1. Dinklage, A., Fischer, R., and Svensson J., Fusion Sci. Technol. 46, 355-364 (2004).

2. Wald, L., IEEE Transactions on Geoscience and Remote Sensing 37, 1190-1193 (1999).

3. D' Antona, G., Cirant, S. Farina, D. et al., "Algorithms for the Control of NTM by Localized ECRH. Principles and Requirements of the Real Time Diagnostics and Control System" this conference, AIP Conference Proceedings , 2008

4. Gregory, P., Bayesian Logical Data Analysis for the Physical Sciences, Cambridge: Cambridge Univ. Press, 2005.

5. Sivia, D. S., and Skilling, J., Data Analysis: A Bayesian Tutorial, Oxford: Oxford Science Publications, $2^{\text {nd }}$ Edition, 2006.

6. Shannon, C.E., Bell Syst. Techn. J. 27, 379 (1948).

7. Challoner, K., Verdinelli, I., Stat. Sci. 10, 273 (1995).

8. Fischer, R., Wendland, C., Dinklage, A., et al., Plasma Phys. Contr. Fusion 44, 1501-1519 (2002).

9. Fischer, R., Dinklage, A., and Pasch, E., Plasma Phys. Contr. Fusion 45, 1095-1111 (2003).

10. Svensson, J., Dinklage, A., Geiger, J., et al., Rev. Sci. Instrum. 75, 4219-4422 (2004).

11.Dinklage, A., Beidler, C.D., Fischer, R., et al. "Integrated Interpretative Transport Modelling" in $31^{s t}$ EPS Conference on Plasma Physics, edited by P. Norreys and H. Hutchinson, Geneva: European Physical Society, 2004, pp. O.2.03.

12.Fischer, R. et al. Phys. Rev. E 61, 1152 (2000).

13.Dose, V. et al. Nucl. Fusion 41, 1671 (2001).

14. Fischer, R., "Bayesian Experimental Design" in Bayesian Inference and Maximum Entropy Methods in Science and Engineering, edited by S. C. Schmidt et al., AIP Conference Proceedings 735, New York: American Institute of Physics, 2004, pp. 43-61.

15. Dreier, H., Dinklage, A., Fischer, R., et al., Rev. Sci. Instrum. 77, 10F128-10F131 (2006).

16.Dinklage, A., Maaßberg, H., Preuss R., et al Nucl. Fusion 47, 1265-1273 (2007).

17.v. Toussaint, U, Gori, S., Dose, V., Neural Networks 19 1550-1557 (2006). 